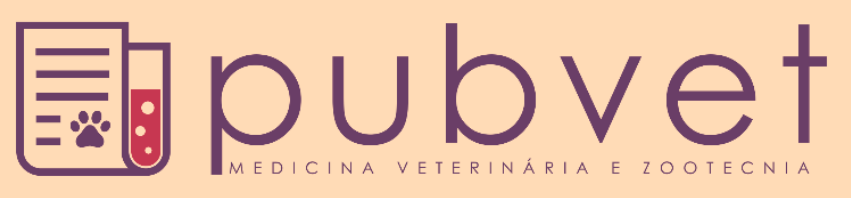

https://doi.org/10.31533/pubvet.v14n5a568.1-5

\title{
Fibrossarcoma em cão Rottweiler: relato de caso
}

\author{
Milena Batista Carneiro ${ }^{1 *} \bullet$, Celia Yelimar Palmero Quintana ${ }^{10}$, Ingrid Carvalho Andrade ${ }^{2} \bullet$, \\ Beatriz Zago Lupepsa ${ }^{3}$, Pollyana Abreu Campos da Cruz $^{3}$, Larissa Teixeira da Silva Fonseca ${ }^{3}$, \\ Greice Nascimento Pires ${ }^{2} \odot$
}

${ }^{1}$ Professora da Universidade Federal do Rio de Janeiro - Campus Macaé, departamento de Patologia. Macaé - RJ Brasil. ${ }^{2}$ Acadêmica de Medicina da Universidade Federal do Rio de Janeiro - Campus Macaé. Macaé - RJ Brasil.

${ }^{3}$ Acadêmica de Enfermagem e Obstetrícia da Universidade Federal do Rio de Janeiro - Campus Macaé. Macaé - RJ Brasil. *Autor para correspondência, E-mail: milenabatistacarneiro@gmail.com

Resumo. O presente estudo tem como objetivo, relatar um caso de fibrossarcoma em paciente canino, idoso, que foi submetido ao exame necroscópico em junho de 2018 após óbito natural. Análises macroscópica e microscópica dos achados patológicos foram realizadas por visualização dos órgãos e por processamento histopatológico das amostras coletadas. Macroscopicamente, foi observada uma massa tumoral pélvica de aproximadamente $15 \mathrm{~cm}$, hipertrofia cardíaca com presença de parasitas na luz ventricular, pulmões congestos e com nodulações, hepatomegalia e atrofia renal. A análise histológica da massa tumoral primária, foi compatível com um fibrossarcoma, com a maioria das células de morfologia fusiforme, com focos de hemorragia e necrose no centro do tumor, alternando com regiões de reação desmoplásica, atipias nucleares e nucléolos proeminentes. Também se verificou, na microscopia, parênquima pulmonar edemaciado com hemorragia difusa, congestão vascular, aumento de espessura dos septos alveolares e nódulos pulmonares sugestivos de lesões metastáticas, assim como fígado com capilares sinusóides congestos e hemorragia difusa no parênquima hepático com focos de necrose de coagulação. O fibrossarcoma é um tumor de origem mesenquimal, composto por fibroblastos transformados, em um fundo de colágeno. Ele ocorre em todas as espécies, porém é mais frequente em cães e gatos. Em cães, manifesta-se normalmente de forma solitária e em indivíduos mais velhos, com média de idade de 9,7 anos. A maioria dos patologistas descreve o quadro histológico do fibrossarcoma como padrão de "espinha de peixe". Habitualmente, é um tumor bastante invasivo, dificultando o tratamento cirúrgico por não permitir ampla margem de excisão, e, sua clínica se manifesta tardiamente, dificultando ainda mais seu diagnóstico e tratamento, como ocorreu no caso relato, em que o paciente veio à óbito subitamente. Deste modo, a análise histopatológica e citopatológica continuam sendo padrão ouro dentre os métodos diagnósticos iniciais para se confirmar a presença do tumor, destacando a importância da aplicação desses métodos de estudo, na determinação das características morfológicas de diferentes tipos de lesões, bem como suas formas de apresentação, cujo diagnóstico pode ser confirmado com outros métodos diagnósticos.

Palavras chave: cão, fibrossarcoma, neoplasia, patologia

\section{Fibrosarcoma in Rottweiler dog: case report}

Abstract. The present study aims to report a case of fibrosarcoma in an elderly canine patient who underwent necroscopic examination in June 2018 after natural death. Macroscopic and microscopic analyzes of the pathological findings were performed by organ visualization and histopathological processing of the collected samples. Macroscopically, a pelvic tumor mass of approximately $15 \mathrm{~cm}$, cardiac hypertrophy with 
parasites in the ventricular lumen, congested and nodular lungs, hepatomegaly and renal atrophy were observed. Histological analysis of the primary tumor mass was compatible with a fibrosarcoma, with most cells of fusiform morphology, with foci of hemorrhage and necrosis in the center of the tumor, alternating with regions of desmoplastic reaction, nuclear atypia and prominent nucleoli. Also, microscopy revealed swollen pulmonary parenchyma with diffuse hemorrhage, vascular congestion, thickening of the alveolar septa and pulmonary nodules suggestive of metastatic lesions, as well as liver with congested sinus capillaries and diffuse hemorrhage in the hepatic parenchyma with foci of coagulation necrosis. Fibrosarcoma is a tumor of mesenchymal origin, composed of transformed fibroblasts, in a collagen background. It occurs in all species, but is more common in dogs and cats. In dogs, it usually manifests in solitary form and in older individuals, with a mean age of 9.7 years. Most pathologists describe the histological picture of fibrosarcoma as a fishbone pattern. It is usually a very invasive tumor, making surgical treatment difficult because it does not allow a wide margin of excision, and its clinic manifests itself late, making diagnosis and treatment even more difficult, as in the case reported here, in which the patient suddenly died. Thus, histopathological and cytopathological analysis remain the gold standard among the initial diagnostic methods to confirm the presence of the tumor, highlighting the importance of applying these study methods in determining the morphological characteristics of different types of lesions, as well as their forms, whose diagnosis can be confirmed with other diagnostic methods.

Keywords: dog, fibrosarcoma, neoplasia, patology

\section{Fibrosarcoma en perro Rottweiler: reporte de caso}

Resumen. El presente estudio tiene como objetivo informar un caso de fibrosarcoma en un paciente canino anciano, sometido a un examen necroscópico en junio de 2018 después de una muerte natural. Los análisis macroscópicos y microscópicos de los hallazgos patológicos se realizaron mediante la visualización de los órganos y procesamiento histopatológico de las muestras recolectadas. Macroscópicamente, se observó una masa tumoral pélvica de aproximadamente $15 \mathrm{~cm}$, hipertrofia cardíaca con parásitos en la luz ventricular, pulmones nodulares y congestionados, hepatomegalia y atrofia renal. El análisis histológico de la masa tumoral primaria fue compatible con un fibrosarcoma, con la mayoría de las células de morfología fusiforme, con focos de hemorragia y necrosis en el centro del tumor, alternando con regiones de reacción desmoplásica, atipias nucleares y nucleolos prominentes. Además, la microscopía reveló parénquima pulmonar inflamado con hemorragia difusa, congestión vascular, engrosamiento de los tabiques alveolares y nódulos pulmonares sugestivos de lesiones metastásicas, así como hígado con capilares sinusales congestionados y hemorragia difusa en el parénquima hepático con focos de necrosis de coagulación. El fibrosarcoma es un tumor de origen mesenquimatoso, compuesto de fibroblastos transformados, en un fondo de colágeno. Ocurre en todas las especies, pero es más común en perros y gatos. En perros, generalmente se manifiesta como tumores únicos, e en individuos mayores, con una edad media de 9.7 años. La mayoría de los patólogos describen la imagen histológica del fibrosarcoma como un patrón de "espina de pescado". Por lo general, es un tumor muy invasivo, lo que dificulta el tratamiento quirúrgico porque no permite un amplio margen de escisión, y su clínica se manifiesta tarde, lo que hace que el diagnóstico y el tratamiento sean aún más difíciles, como en el caso informado aquí, en el que el paciente murió repentinamente. Por lo tanto, el análisis histopatológico y citopatológico sigue siendo el estándar de oro entre los métodos de diagnóstico iniciales para confirmar la presencia del tumor, destacando la importancia de aplicar estos métodos de estudio para determinar las características morfológicas de los diferentes tipos de lesiones, así como sus variaciones, cuyo diagnóstico puede confirmarse con otros métodos de diagnóstico.

Palabras clave: perro, fibrosarcoma, neoplasia, patología 


\section{Introdução}

O fibrossarcoma é um tumor de origem celular mesenquimal, composto por fibroblastos malignos em um fundo de colágeno. Existem dois tipos principais, o primário e o secundário. $\mathrm{O}$ fibrossarcoma primário é uma doença maligna fibroblástica que produz quantidades variáveis de colágeno, podendo ser central, com o surgimento no interior do canal medular de crescimento lento, ou periférico, decorrente do periósteo. Já o fibrossarcoma secundário do osso surge de uma lesão preexistente ou após radioterapia para uma área de tecido ósseo ou mole (Mopper, 1953; Silva et al., 2011). Esse tipo de tumor ocorre em todas as espécies, sendo mais frequente em cães e gatos. Em cães, manifesta-se normalmente de forma solitária e em indivíduos mais velhos, com média de 9,7 anos (Magalhães et al., 2015). Ocasionalmente pode ser encontrado em animais jovens e até menores de seis meses de idade. Não há predisposição por raça ou sexo (Hauck, 2003). A causa do fibrossarcoma ainda não é conhecida, embora acredita-se que mutações genéticas possam ter um papel importante, incluindo defeitos genéticos como perda de alelos, mutações pontuais e translocações cromossômicas (Mopper, 1953; Silva et al., 2011).

Macroscopicamente os fibrossarcomas são irregulares, de coloração branco acinzentado, de tamanho variável e consistência firme (Thomson, 1990). Por serem altamente vascularizados é comum a presença de hemorragias e necrose, apresentando-se ulcerados (Ribeiro et al., 2011). O grau de malignidade é avaliado de acordo com a capacidade de infiltração e indiferenciação celular, número de mitoses, células multinucleadas com dois ou três núcleos. As células apresentam-se entrelaçadas ou arranjadas num padrão de redemoinho, exibindo alto pleomorfismo, variando desde células fusiformes indiferenciadas, com núcleos redondos a ovóides, até células alongadas entremeadas com feixes que se parecem com o tecido conjuntivo imaturo associado a presença de células dispostas em grupos paralelos entre si (Ribeiro et al., 2011).

O objetivo do estudo é relatar um caso de fibrossarcoma em paciente canino que foi submetido à necropsia, após óbito natural, com posterior análise microscópica para confirmação do diagnóstico.

\section{Relato de caso}

Cão, idoso, macho, da raça Rottweiler foi encaminhado para exame necroscópico após morte natural súbita. O mesmo possuía história pregressa de tumor pélvico associado a edema, assim como, perda de movimento e sensibilidade do membro posterior direito. Antes do óbito, o cão não apresentava alterações na micção ou evacuação.

Diante deste quadro clínico, realizou-se o exame necroscópico em que foram encontradas algumas alterações anatomopatológicas importantes para a determinação da causa mortis. Tais achados foram analisados tanto macroscopicamente, no momento da necrópsia, quanto microscopicamente, por meio de biópsias coletadas dos órgãos avaliados, que passaram por todas as etapas de processamento histopatológico para a confecção de lâminas.

Os achados macroscópicos incluem: 1) Massa tumoral pélvica, próxima ao rim esquerdo, aderida à bexiga e à coluna vertebral, com $15 \mathrm{~cm}$ de diâmetro, de consistência firme, irregular, mal delimitada, friável e vascularizada; 2) Hipertrofia cardíaca concêntrica com presença de parasitas da espécie Dirofilaria immitis no ventrículo esquerdo; 3) Pulmões difusamente congestos bilateralmente, apresentando múltiplos nódulos de tamanhos distintos e de consistência variada, sugestivos de metástase; 4) Hepatomegalia e presença de lesões circulares em cápsula hepática, também sugestivos de metástase; 5) Rim esquerdo atrófico devido a provável compressão pelo tumor pélvico.

Já os achados microscópicos encontrados após estudo histopatológico foram: 1) Lesão tumoral pélvica sugestiva de fibrossarcoma, com predominância de células fibroblastoides, focos de hemorragia e necrose, alternando com regiões de reação desmoplásica, atipias nucleares e nucléolos proeminentes; 2) Parênquima pulmonar com edema generalizado e hemorragia difusa, congestão vascular e aumento de espessura dos septos alveolares, macrófagos alveolares com hemossiderina e espessamento da pleura visceral com infiltrado inflamatório local, além de nódulos pulmonares delimitados por cápsula, com características similares às da lesão tumoral pélvica e sugestivo de lesões metastáticas; 3 ) Fígado com capilares sinusóides congestos, hemorragia difusa no parênquima hepático e foco de necrose de coagulação. Tais alterações estão apresentadas na Figura 1. 


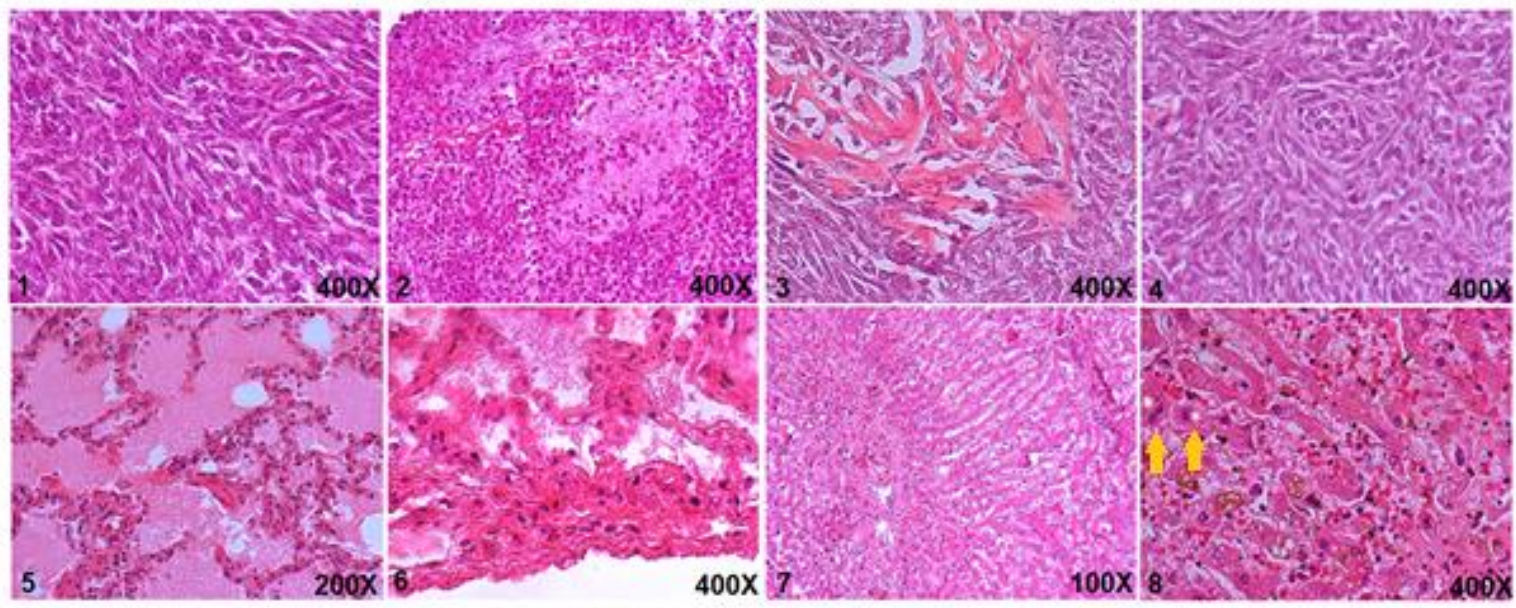

Figura 1. Achados microscópicos: 1) Células tumorais com formato fíbroblastóide; 2) Área de necrose e hemorragia, no centro do tumor; 3) Reação desmoplásica, onde se podem observar células tumorais entremeadas com fibras de colágeno de cor rosa; 4) Pleomorfismo nuclear e nucléolos proeminentes; 5) Edema pulmonar generalizado, espessamento do septo alveolar e congestão vascular; 6) Detalhe dos alvéolos edemaciados e do espessamento da pleura visceral; 7) Infiltrado inflamatório e dilatação dos sinusóides hepáticos; 8) Sinusóides hepáticos congestionados, bem como necrose de alguns hepatócitos na metade superior da imagem. Além disso, pode-se observar a presença de dois hepatócitos binucleados ( $)$, assim como a presença de pigmento biliar (de cor acastanhada) no interior de alguns hepatócitos no canto inferior esquerdo da microfotografia.

\section{Discussão}

Pelas análises macro e microscópicas do caso relatado e dos estudos acerca do fibrossarcoma é possível sugerir que o paciente em questão apresentava uma forma primária desta patologia, provavelmente aderida ao periósteo, de acordo com a localização anatômica em que a massa foi identificada (Borba et al., 2003). Macroscopicamente, apresenta coloração branco acinzentado, semelhante a descrição feita por Thomson (1990). Já em relação à microscopia, a maioria dos patologistas descreve o quadro histológico do fibrossarcoma como padrão de espinha de peixe, um entrelaçamento de fibroblastos fusiformes em um fundo de colágeno, com formas bem diferenciadas, núcleos profundamente corados e uma produção exagerada de colágeno. Este padrão de pleomorfismo, hipercromasia e células multinucleadas são achados comuns ao fibrossarcoma e geralmente confirmam o diagnóstico (Thomson, 1990). Todas as características citadas acima para diagnóstico histopatológico do fibrossarcoma foram encontradas no caso relatado.

O caso também apresenta relato de hipertrofia cardíaca e acometimento pelo parasita Dirofilaria immitis, agente causador da doença Dirofilariose. Este parasita costuma alojar-se no coração e nas artérias pulmonares. Durante a evolução da enfermidade ocorre febre, hematúria, hepatomegalia e esplenomegalia, prurido, aparecimento de nódulos cutâneos e até convulsão (Santos et al., 2001). A Dirofilariose é uma zoonose parasitária não contagiosa complexa, de evolução crônica, apresentandose de forma clínica ou subclínica que acomete principalmente o cão doméstico, gato e várias espécies de animais silvestres. A maioria dos animais acometidos não apresentam sintomas da doença até que a mesma atinja um estágio avançado, sendo assim uma fonte de contágio (Santos et al., 2001). O cão relatado não apresentou alteração na micção, assim como não manifestou hematúria macroscópica. Entretanto, o mesmo tinha uma importante hepatomegalia com presença de capilares sinusóides congestos, hemorragia difusa no parênquima hepático e focos de necrose de coagulação.

Notou-se também a presença de focos nodulares em pulmões e em cápsula hepática que, ao serem submetidos à análise histopatológica, apresentaram o mesmo tipo celular do tumor, indicando, assim, focos metastáticos do fibrossarcoma. Habitualmente os fibrossarcomas são bastante invasivos, o que dificulta o tratamento cirúrgico por não permitir ampla margem de excisão. $\mathrm{O}$ desenvolvimento de metástases é raro ou tardio, ocorrendo em menos de $10 \%$ dos animais com tumores isolados (Birchard 
\& Sherding, 2008); porém, por esta ser uma neoplasia altamente infiltrativa e recidivante, pode fazer metástases em linfonodos, pulmões e vértebras principalmente (Mopper, 1953; Silva et al., 2011).

\section{Conclusão}

Diante da exposição do caso, é possível observar que o fibrossarcoma é um tumor silencioso, com sintomas que, na maioria dos casos, demoram a se manifestar e que são mais usualmente encontrados em pacientes idosos, como em cães na sua segunda década de vida. É uma neoplasia maligna de difícil abordagem cirúrgica e cujo diagnóstico deve ser precoce e preciso. Deste modo, a análise citológica e histopatológica são os principais métodos diagnósticos iniciais para se confirmar a presença do tumor. No caso relatado, devido ao quadro avançado da doença apresentado pelo paciente, o mesmo não teve a oportunidade de receber um tratamento preconizado, vindo à óbito de forma súbita e natural.

\section{Referências bibliográficas}

Birchard, S. J., \& Sherding, R. G. (2008). Manual Saunders: clínica de pequenos animais. In Editora Roca (Vol. 3).

Borba, P. P., Fiod, N. J., Gouveia, G. C., Nascimento, M. F., \& Rezende, J. F. (2003). Fibrossarcoma epitelióide esclerosante: relato de caso. Revista Brasileira de Cancerologia, 49, 221-225.

Hauck, M. (2003). Feline injection site sarcomas. The Veterinary Clinics of North America. Small Animal Practice, 33(3), 553-557.

Magalhães, G. M., Santilli, J., Calazans, S. G., Nishimura, L. T., de Amorim Cerejo, S., \& Dias, F. G. G. (2015). Fibrossarcoma primário em intestino delgado de cão-Relato de caso. Brazilian Journal of Veterinary Medicine, 37(2), 145-148.

Mopper, C. (1953). Primary fibrosarcoma of the skin. Journal of the American Medical Association, 152(7), 570-572.

Ribeiro, F. P., Hamzé, A. L., Pacheco, A. M., Trentin, T. C., Lot, R. F. E., Friolani, M., Dias, L. G. G. G., \& CABRINI, T. M. (2011). Fibrossarcoma em cão - Relato de caso. Revista Centífica, 9(16), 16.

Santos, L. A. C., Silva, F. C., \& Montanha, F. P. (2001). Dirofilariose em pequenos animais - Revisão de literatura. Revista Científica Eletrônica de Medicina Veterinária, 9(17), 1-6.

Silva, M. M. V, Crivelenti, L. Z., Momo, C., \& Honsho, D. K. (2011). Fibrossarcoma uretral primário em cadela. Arquivo Brasileiro de Medicina Veterinária e Zootecnia, 63, 1353-1358.

Thomson, R. G. (1990). Patologia veterinária especial (Vol. 1). Editora Manole, São Paulo, Brasil.

Recebido: 15 de dezembro, 2019.

Aprovado: 20 de janeiro, 2020 .

Disponível online: 6 de junho, 2020

Licenciamento: Este artigo é publicado na modalidade Acesso Aberto sob a licença Creative Commons Atribuição 4.0 (CC-BY 4.0), a qual permite uso irrestrito, distribuição, reprodução em qualquer meio, desde que o autor e a fonte sejam devidamente creditados. 